\title{
The integration of 'foreign' technologies into sporting practice
}

In 1999 I was employed as a gymnastics coach in the USA, and one of the gymnasts I coached was trying to obtain a college scholarship. So I enrolled a device foreign to the gymnastics environment, a video camera, and brought it to the gymnasium to film her performing her routines. Following this exercise, she made numerous copies of the videotapes and posted each tape to a different college. In this example the enrolment of the video camera allowed the performance of the gymnast to be made mobile. Indeed, the performance was transformed from a live moment in time to one that could be watched as many times as desired, through the enrolment of a video camera and tape. The video camera and tape acted as intermediaries, in allowing the gymnast's performance to be captured and mobilised.

I am no longer a gymnastics coach, but I continue to be a gymnastics fan. As a fan, I particularly enjoy the existence of YouTube, in allowing me to view routines that I would not otherwise be able to watch from my remote location in New Zealand, where elite gymnastics is rarely televised. But my searches on YouTube have revealed more than merely elite routines; I also come across numerous YouTube sites by athletes such as the one I coached in 1999, set up to display their performances in order to entice colleges to provide them with scholarships. So I am aware that the process of college selection has altered since 1999. In one sense it is the same, since the tool of video is still utilised to stabilise the routine and to make it mobile. However, it is also fundamentally different, as the necessity of posting out tapes is no longer required. College selectors can instead simply trawl through applicants' YouTube sites in order to view their footage and use it to make their decisions. The laborious process of sending out individual tapes to each college no longer needs to take place.

These examples illustrate some of the central concepts of this chapter. First, the introduction of foreign technologies into sporting environments can act to change sporting processes in a variety of ways. The presence of YouTube has changed the way that athletes can now make their performances visible to college selectors. 
At the same time, the basic application process remains the same, and both the videotape-and-postal-service method and YouTube act in the same way, as intermediaries that make the performances of the gymnasts mobile to the selectors.

As in the above examples, sporting performances and processes can be strongly influenced by technology and equipment that are not considered a standard part of sport. This chapter focuses on the introduction of 'non-sport' technologies into the sports training environment. It includes two case studies: a study of the use of global positioning systems (GPS) in Australian rules football (AFL) and an examination of the use of technologically constructed hypoxic environments (TCHEs), commonly known as altitude chambers. The previous chapter introduced the concept of enrolment, an ANT idea employed to determine how an actant comes to be part of an actor-network, which is also drawn upon in this chapter. In particular, both cases highlight the difficulty of creating equal opportunities for enrolment. In the case of GPS, the difficulty concerns equalities between all teams in the league, while for TCHEs the inequalities are reflective of global economic disparities.

This chapter also continues the theme of sport as a socio-technical network, which runs throughout the book. The previous chapter argued that an athlete's network contains a myriad of factors that influence whether athletes utilise new enhancements, and this chapter expands on this notion by considering how it is that technologies can act to produce particular outcomes. GPS units are found to act as intermediaries, mediators or as methods of surveillance, all of which have very different outcomes. Also included in the understanding of a socio-technical network is the argument that it is not merely physical objects and humans that make it up, but that it has a range of ideas 'folded' (Latour and Venn, 2002) within it. The incorporation of historical and current discourses as part of the network is considered through the case of TCHEs. With TCHEs I argue that the range of discourses connected to an understanding of these technologies together with an athlete's individual network strongly influences enrolment and use of the devices.

\section{The ANT conception of power}

One focus of this chapter is the notion of 'technologies as actants' within the socio-technical network that makes up sport. The chapter therefore illustrates how examining the network reveals the power relations within it, often through the production or prevention of action. 
The ANT perspective conceives of power as an action or effect that occurs through interactions within the network (Latour, 1996; Law, 1992; Matthewman, 2011). Through understanding power as an effect, ANT shows how both humans and non-humans can equally cause action but also, more importantly, that action occurs through humans and non-humans working together. For example, Latour (2009) describes how the statement 'guns kill people' is flawed in the sense that it is neither the individual gun nor the individual person that performs the killing, but a third actant, what Latour refers to as a 'citizen-gun' that causes the action. ANT theorists emphasise that agency is shared between humans and non-humans and that it is the way the actants assemble as a network that creates the action. As such, power flows through the network. The gun and the person do not necessarily have to assemble to kill someone, but killing is one effect that the network can produce.

In acknowledging that effects are produced because of the make-up of the network, ANT is interested in following the strands of the network to understand the way that local agency is enacted at every level of an apparent hierarchy (Matthewman, 2011). This notion resembles Foucault's conception of power, which described it as existing in a 'capillary-like network' where every point in the network can affect other points in a flat, rather than hierarchical, structure (Markula and Pringle, 2006, p. 36). Latour emphasises that it is the size, shape and scope of the network that will determine what is produced. Different kinds of actor-networks, or assemblages, will produce different kinds of effects (Edwards and Nicoll, 2004; Kerr, 2014).

As noted in the Introduction, Latour (2005) argues for the existence of what he terms intermediaries and mediators. He describes intermediaries as non-controversial actants that behave in a particular way each time (Bencherki, 2012; Kerr, 2014; Latour, 2005). By contrast, a mediator is an actant that behaves unpredictably and disrupts the network. For example, in the context of sport, a competitive cyclist consists of a particular network assembled from a person and a bicycle plus numerous other components, such as a helmet and skin-tight suit. If the bicycle propels the cyclist at a fast rate, it is behaving as an intermediary as expected. However, the cyclist may be prevented from cycling as fast as desired through the presence of mediators. In cycling, hair acts as a mediator through creating wind friction which slows the cyclist down, so intermediaries such as shaving and an aerodynamic helmet are introduced to suppress the hair.

These distinctions are significant, as mediators demonstrate how it is that non-humans can act to disrupt the desired action, holding power through 
causing unexpected outcomes, while intermediaries hold power through ensuring action and at times suppressing mediators to perform as desired. However, Latour's central point is that in all contexts, humans work together with non-humans in order to function, and that therefore both the human and non-human can cause action: hence the emphasis on seeing the world as networks, not individual components. A cyclist is an assemblage of human and non-human actants that act together to travel faster than either can alone. Agency is shared equally between the human and non-human actants (Bencherki, 2012; Latour 2005, 2009).

Such a view reverses the standard sociological conception of agency. For Actor-Network Theorists, agency is produced through a heterogeneous network rather than individually. In the cyclist example above, agency is produced through the human cyclist, bicycle and other associated non-humans such as helmets, shaving and skin-tight suit, which, depending on the properties of each and how they assemble, will determine the speed at which the cyclist moves.

This chapter explores the notion that technologies act as mediators or intermediaries through the case study of the GPS units introduced into AFL. Following this, the example of hypoxic chambers (TCHEs) is used to understand the breadth of what makes up a network and its ability to act. TCHEs are shown to have a variety of ideas 'folded' (Latour and Venn, 2002) within them that make their use both understandable and also controversial.

\section{Case study: the use of global positioning systems (GPS) in Australian rules football}

The introduction of GPS units into the Australian Football League (AFL) provides fruitful study for understanding how different actor-networks can produce particular effects, how technologies can act within the network and how surveillance can be enacted through new technologies. The cases thus far have focused on the issue of enrolment. In this case enrolment is only a small part of the discussion, since GPS units have been enrolled in Australian Football League for several years. Instead, this section focuses on GPS units as actants.

AFL is one of the most popular sports in Australia, and boasts the largest number of attendees of any sports: 6.5 million in 2011 (Foreman, Deegan and 
Wigley, 2012). The AFL was an early adopter of GPS, having used it within the league since 2005 (Foreman, Deegan and Wigley, 2012; Wisbey, Rattray and Pyne, 2010). AFL has possibly the highest uptake of GPS of any sport (Aughey, 2011).

The GPS units used in the league consist of small units worn on the backs of players that transmit data to satellite and then to a computer or other device able to receive the satellite signals. Foreman, Deegan and Wigley (2012, pp. 1-2) describe the exact details:

Athletes wear a small (approximately $80 \times 50 \mathrm{~mm}$ ) transmitter on their upper back in a purpose-built supportive harness ... which measures and analyses data from sensors, including multi-axis accelerometers and heart rate monitors ... The data is collected and broadcast simultaneously to the remote computer for 'real time' analysis. Sports scientists can therefore view real time performance and tactical data during matches or training sessions. From the analysis of the data, the coaching team can make tactical changes, analyse player effort or manage player rehabilitation.

The use of GPS units allows a significant amount of data to be collected that can be used to assist sport scientists and coaches with maximising athlete output throughout the game (Foreman, Deegan and Wigley, 2012). During the play, the data generated through the GPS systems includes player speeds, player intensity, player workload, player rotation and player movement patterns (Wisbey, Rattray and Pyne, 2010). In the training environment GPS can be used to evaluate athlete strength and conditioning and to set programmes for tactical and strategic analysis, for play rehabilitation and for injury management (Foreman, 2009). Many of these applications are essentially ways of making the internal workings of the body, or of a complex game, easily visible to scientists who are employed to improve sporting performance. So, unlike technologies that act directly to improve athletic performance, these act indirectly, needing the translation of a scientist in order to be useful. The scientist is necessary for interpreting the data provided by the GPS through comparing and contrasting it with expected norms and other previous information in order to inform coaches about which players are operating at their best and therefore assist the coach with making decisions within the field of play. It is the networked arrangements between the GPS units, the scientists and the coaches that make the GPS units particularly useful within the game and not simply the technology itself. 
GPS units were introduced somewhat slowly into AFL competitions. In 2005 and 2006 AFL clubs were permitted access to GPS units but were allowed to use them for only five players per team over ten games per year (Le Grand, 2007). At this point only half the teams in the league used the units (Wisbey, Rattray and Pyne, 2010). In 2007 all sixteen AFL clubs lobbied to lift the restrictions. Initially, the league argued against this for a number of reasons. First, they were concerned about the issue of equality. The units cost around $\$ 4000$, so were expensive for clubs to buy (Foreman, 2009), and the league were anxious that richer clubs would therefore be at an advantage in being able to purchase more of the units than the poorer clubs. Given the need for data analysts to interpret the data, as discussed above, the need to employ them was an extra cost for clubs to bear. Another issue of concern was that of athlete safety.

As the units are worn on the upper back of the players, there is a possibility that they could cause injuries during tackles (Le Grand, 2007). While the problem here could be understood as being introduced by the technology itself, in reality it is an issue caused by the particular network of sports rules, humans and technologies. Other sports have not enrolled the GPS units because their particular network includes elements that would make it unsafe for players to wear the units. For example, rugby union has not enrolled GPS units because the sport includes tackles as a significant part of its network.

Finally, the league was also concerned that the increased GPS data would allow teams to circumvent newly introduced rules brought in to slow the game down (Le Grand, 2007). However, these concerns were not considered sufficient to prevent their use. Therefore, the restrictions were lifted, and now every team in the league uses GPS (Wisbey, Rattray and Pyne, 2010).

\section{Does GPS provide the promised benefits?}

It appears to be agreed that the GPS units do provide a large quantity of data that many teams find useful (Aughey, 2011). In that sense, these units are intermediaries, acting as expected every time they are used. Aughey (2011, p. 302) describes how the use of the units has changed and extended over the years since their adoption in 2005, moving 'from general descriptive work on player movement in matches to the analysis of fatigue, comparison of real-time to post-game analysis of data, and comparisons among playing levels. This quote suggests that GPS units act through fulfilling their desired role of providing extensive information to players, coaches and scientists. 
Foreman's (2009) analysis found GPS to be particularly useful in three different areas. First, GPS can maximise player fitness through monitoring player fatigue levels and thereby providing information to coaches that allows players to be rotated off the field during competition, and through confirming overall fitness levels so coaches can prepare individual training programmes to maximise individual fitness. Second, GPS can provide information to assist coaches with tactical decisions, such as showing them precisely where all the players are during a live match. Finally, GPS can assist with rehabilitation by providing feedback on whether a player is over- or under-loading their training when they are returning from injury.

Aughey (2011) goes on to describe the usefulness of the GPS data in comparative terms. He argues that GPS data has been useful in comparing expenditure of energy 'across a match, between matches, between levels of competition, and between types of matches' (Aughey, 2011, p. 303). Comparative data of this nature is described as providing greater understanding of the demands made on players, thereby allowing coaches to configure training sessions to work on areas of weakness revealed by the data (Wisbey, Rattray and Pyne, 2010).

Foreman, Deegan and Wigley (2012) investigated thoroughly the question of whether GPS units are useful devices. One finding from their study was that the accuracy of the GPS units is questionable. They point out that, although the manufacturer's website claims that the units have a high degree of accuracy, with a margin of error of only two to four per cent, other independent studies reveal inaccuracies of up to 140 per cent (see, for example, Aughey and Falloon, 2010). They further argue that these margins are for straight-line sprinting, which is rarely the case in AFL as the game requires players to change direction constantly, in line with what is happening in the game. Therefore, they conclude that although GPS units are an effective tool to use during training sessions, their accuracy prevents them from being useful for the purpose of tracking player movement during the game. In this example the GPS units are essentially acting as mediators, rather than providing the exact positions of the players as desired, which would mean the units were acting as intermediaries.

In addition to the accuracy issue, in keeping with the ANT perspective, the GPS units cannot be considered without examining the socio-technical network in which they operate. This chapter will now discuss the network that surrounds the GPS units with a view to determining whether the units can 
be viewed as intermediaries when examined as part of an actor-network. In all the examples below, it is found that the GPS units can only do their job as intermediaries if the appropriate network is in place. In some cases, it is found that an aspect of the existing network acts as a mediator in preventing the GPS units from acting.

One of the major considerations when using GPS is the analysis of the data provided. Unfortunately, at the current time, there is no technology that can automate the data analysis: significant advances in the field of computer vision would be required for it to be achieved (pers. comm., Richard Green, 2013). Therefore, clubs have created different kinds of networks to solve this problem. The richer clubs have opted to employ several skilled specialists to analyse the data and edit it into a form useful for coaches and athletes. By contrast, those clubs that lack sufficient economic capital as part of their network must instead resort to measures such as the one adopted by Richmond, who employed a student performing work experience to 'crunch' the data (Le Grand, 2007). In this respect, the GPS devices require the addition of a human in order to allow them to act as intermediaries.

Further, different ways of using the data need more specific expertise. For example, one useful tactic is to synchronise the GPS data with game or training time to allow coaches to correlate what is happening on the field with the GPS data. Foreman, Deegan and Wigley (2012) found that it was unexpectedly difficult to align the two as they have different timestamps, and therefore that very few clubs were able to afford to access the specific expertise needed to utilise this aspect of the programme. It thus remained a feature that was not enrolled, and again the GPS was unable to act unless the network included this highly specific expertise.

Similarly, Foreman, Deegan and Wigley (2012) described one of the most heavily advertised features of the GPS as not being used: the ability to track the structure, movement and space of the team. They argued that this is impossible to achieve unless clubs own an individual GPS unit for every player and, because of the cost very few clubs can afford this. Therefore, this is another feature that remains not enrolled, with the units unable to act.

The network can also contain features that actually prevent technologies from acting, as is the case with the various stadiums that clubs use. If a stadium includes a closed roof, the GPS signals are unable to get through, rendering the technology completely useless. As sports journalist Le Grand (2007, p. 43) 
describes, a number of teams are affected by this: GPS 'doesn't work under the closed roof of the Telstra Dome. This puts clubs such as St Kilda, the Western Bulldogs, Essendon, Carlton and the Kangaroos, who all use Telstra Dome as a home venue, at a disadvantage.' However, Le Grand (2007) also notes that one of these clubs, the Bulldogs, found a way around it by partnering with the Victorian University of Technology, who provided an alternative way for them to receive some of the data that would normally be received through the GPS units. In this last example, the job of the GPS was delegated to an alternative technology in order to create the necessary action, but it is ironic that this is necessary, given that the data provided is one of the main reasons for using the GPS units.

GPS units have also been found to include features that are simply not useful in the AFL scenario. One such feature is the ability to compare a player's intensity during a game with their intensity during training. While on the surface this sounds useful, the goal of an AFL training session is not necessarily to mirror the intensity experienced in a game, so this feature is not useful (Foreman, Deegan and Wigley, 2012). This scenario is similar to the situation in French kayaking described by Trabal (2008). In both cases the designers of the technology, whether boats or GPS devices, approach sport with a different understanding from that of the athletes and coaches who are directly involved. Therefore the requirements of the two groups do not necessarily align and the result is, again, non-enrolment.

All the above examples demonstrate the importance of understanding technology as part of a heterogeneous network if maximum usage of a technology is the desired outcome. When making the decision to allow GPS units to be used, the league considered the units as singular technological items made up of boxes to be worn on players' backs. The league raised concerns about the individual costs of the units and factored this into their decision to allow GPS units, but omitted consideration of the many other components that produce the network of a working GPS unit. As a consequence, the units are not able to act in the way that they were set up for, and the league is losing some of the potential value of the units. Other parts of the network act as mediators in preventing the GPS units from working to their full potential. The GPS units could have been of greater benefit in AFL if the additional parts of the GPS network, such as experts and roofs, had been considered in the planning around the use of the devices. 


\section{Acting through surveillance}

In contrast to the above examples where the GPS units were not able to act, there was one way that the GPS units were able to act very effectively: to provide player surveillance. One of the ways that an ANT perspective differs from others is through the assertion that power is enacted through non-human devices. This point is well illustrated through an examination of the way GPS units act as surveillance units.

One of the most prominent theorists who explored surveillance is one whose work shares many similarities with ANT, Michel Foucault. In previous works (see Kerr, 2014) I have noted that ANT and Foucauldian theory hold a number of similarities, most importantly their understanding of power; indeed, one of the most prominent ANT theorists, John Law, claims that ANT owes a debt to Foucault in this regard (Law, 1992). However, in line with the ANT perspective discussed throughout this book, ANT goes further than Foucault in arguing for the significance of non-humans as actors in the workings of society.

Foucault argued that power reaches bodies through what he terms 'dispositives', also at times translated as 'apparatus', which refers to anything that affects the behaviours, beliefs or views of any living being (Legg, 2011). Prison architecture was one type of apparatus that Foucault described (Hekman, 2009), with Foucault arguing that the famous Panopticon prison design would have a distinct effect in creating self-regulation on the part of the inmates. It is Foucault's concept of power being enacted through a material form that connects strongly with Latour's work (Dorrestijin, 2012). For example, in his book Discipline and Punish, when discussing the Panopticon, Foucault states, 'Power has its principle not so much in a person as in a certain concerted distribution of bodies, surfaces, lights, gazes' (Foucault, 1977, p. 220), thereby acknowledging the way power relations are enacted through material forms.

Within the sporting context, Manley, Palmer and Roderick (2012) utilised the ideas of both Foucault and Latour to demonstrate the way surveillance took place through material forms in their study of a competitive football academy. They described (2012, p. 306) how the academy collected a wide range of data that was used to regulate the athletes' bodies in a very detailed manner:

Control functions took the form of documentation, data or information that was retrieved from a process of video surveillance, human observation and physiological testing. These data were then used to collate knowledge that was 
presented to those further up the academy hierarchies. Fitness tests, physiological tests and review sessions within the football academy aided the shaping and regulation of the athletes' behaviour so they may improve their performance ... The categorisation of the athletes' qualities allowed for the regulation of the minutest detail in relation to performance.

The process described here essentially reduces the athletes' performances to numerical values, or to 'data', for the specific purpose of influencing the athletes. The data was used to situate athlete performance within the parameters of successful performance, which had the effect of ensuring that athletes self-regulated their behaviour in order to meet required standards.

The use of GPS units in AFL echoes this process. In AFL the GPS units were also used for the collection of 'data'. This information could take the form of details around player speed and movements and data about heart rate that is believed to demonstrate how hard a player is working. The information is then utilised primarily by coaches and other officials in order to make decisions. As such, the GPS units act as a way for coaches to increase their level of surveillance of the players. Manley, Palmer and Roderick (2012, p. 308) suggest that such data

can be processed to create a 'digital persona' (Clarke 1994) constituting a 'Superpanopticon', i.e., 'a system of surveillance without walls, windows, towers or guards' ... It is the creation of a 'digital persona' and the collation of data that enabled the coaches and managers to survey their athletes in a range of aspects.

Deleuze (1992) refers to a digital persona as a 'coded body'. Both concepts describe the notion of an electronic body that acts as a different version of a player who also possesses a physical body.

Given the physical presence of the GPS units on the players' backs and the inclusion of data analysts as part of the coaching team, players could not help but be aware of the collection of this data. Foreman, Deegan and Wigley (2012, p. 5) argued that the athletes were aware of the increased surveillance and at times resisted it, stating 'players do not feel comfortable wearing GPS devices as they believe they are not being trusted by coaching staff to perform their on-field role. This remark suggests that players feel as though the coach is using the GPS units to test whether they are working hard enough and resent the fact that coaches feel the need to do this.

Additionally, the increased surveillance was felt outside of traditional training time. Pierik (2013) describes how GPS units were used by coaches to monitor 
their players' fitness during their pre-season break, even when the players were not officially training. However, this practice has recently been banned, owing to the perceived need for players to address their work-life balance through having a complete break from play (Pierik, 2013).

In this final section I argue that the wearing of the GPS units allows coaches to increase their level of control over players through providing additional information about the players' whereabouts and level of exertion. From the coaches' point of view, the GPS unit works as an intermediary in working to provide information that the coaches desire. However, as the ANT perspective points out, the GPS unit cannot be considered in isolation, and so from the players' perspective, the GPS device can disrupt effective performance through providing unwanted information to the coach. In this sense, the GPS is closer to the role of a mediator than an intermediary, as it prevents players from working as effectively as their coaches might hope. This is particularly the case during the pre-season break, when the player may believe he needs time for rest and recuperation, whereas the coach may believe the player should be staying in shape.

The case of the GPS units within the AFL league highlights two of the most important aspects of the ANT view of technology that were introduced in Chapter 1. First, it demonstrates how understanding technology as singular, rather than an actor-network, can be problematic for organisations who want to maximise sporting performance through the use of technology. Through considering the enrolment of only one part of the network (the unit worn on the player's back), the other parts that were necessary for the GPS units to work to full potential were not enrolled and consequently reduced the ability for the GPS units to act as intermediaries. Second, the case highlighted the way that non-humans can act to produce or prevent action. The material properties of the GPS units meant that they were able to provide coaches with genuinely useful information, and within this context the units acted as intermediaries. Indeed, the information the units provided proved so useful for coaches that they then began using the units to survey their athletes and monitor their performance in a way that the athletes did not anticipate. This led to resentment of coaches and the creation of an official ruling to prevent such a high level of surveillance.

I now turn my attention to a very different technological device, where use is decided on an individual rather than league basis, and the benefits of the device are potentially more questionable than the GPS units. 


\section{Case study: the use of technologically constructed hypoxic environments}

In the case described above, the introduction of the GPS units into the AFL was relatively uncontroversial. By contrast, the use of devices that imitate the effects of altitude training, such as technologically constructed hypoxic environments (TCHEs), remains controversial in sport because of the argument that the effects mirror those of doping: in particular, EPO and blood doping. Nonetheless, they remain a legal technology in sport. This section will investigate the legality of TCHEs through the notion of what is 'folded' (Latour and Venn, 2002) in a technology. Like the case of the GPS units, this section again emphasises the significance of viewing technologies as actor-networks. However, where the GPS unit's effectiveness was hampered by lack of support from the necessary network that existed beyond the physical unit, in the case of the TCHEs I will argue that it is the actor-network within the units themselves that produces the controversy. Where for the GPS case I needed to follow the units outwards to the network, in this case I will instead follow the TCHE network inwards. Specifically, I argue that TCHEs have a particular history folded within them, including the decisions made at the 1968 Olympic Games and the apparent natural superiority of long-distance runners from places such as Kenya and Ethiopia, which are at a high altitude. In a way, this approach has some similarities to that of authors such as Magdalinski (2009), who notes that the cultural meanings of technological devices are important for understanding their use. In this chapter I adopt a related though somewhat different approach of following the history of the debate, which I argue has become a significant part of the network that we call a TCHE. I argue that the network also incorporates a simplicity of use and lack of medicalisation that results in athletes feeling more comfortable using the devices.

TCHEs are an interesting case to explore, as they sit at the very edge of technologies permitted for use by the World Anti-Doping Agency (WADA). In 2006 WADA commissioned an inquiry into the use of TCHEs and decided not to place them on the banned list, despite their conclusion that TCHEs met two of the three criteria that would entail banning. WADA describes how a technology that meets two of the three criteria 'may, but is not required to, be added to the Prohibited List' (WADA, 2006, para. 6). 


\section{From altitude training to TCHEs}

Major international research and discussion around the effects of training or competing at altitude began in a significant way with the 1968 Olympic Games, which were held in Mexico City, a city at higher than normal altitude. Kasperowski (2009) reports how the organisers of the games addressed the issue of altitude in their bid in 1963, stating that concerns with competing at altitude had been

artificially created, undoubtedly in good faith, but due to a lack of familiarity with the facts. It is definitely refuted by the various documents that follow ... athletes only require a 3 or 4 day period in order to adapt themselves completely to Mexico City's altitude. (Mexican Olympic Committee, 1963, cited in Kasperowski, 2009, p. 1264)

Currently, it is understood that athletes require four to six weeks in order to adjust to altitude; however, the Mexican Olympic Committee's statement is unsurprising given the lack of research that had been conducted at that time. While there had been some experimentation with overcoming issues associated with altitude in order to climb high mountains, there had been no research undertaken on the effects of medium-level altitude (in the range 1800-3000 metres, with Mexico City at 2300 metres). Consequently, between 1964 and 1968 a large amount of research was undertaken into the effects of altitude in the lead-up to the Games (Kasperowski, 2009).

There were several reasons for the interest in the effects of altitude. First, there were concerns over the health of the athletes in competing at altitude, with some citing risks of black-outs and even death. Second, there was a concern with inequality (Kasperowski, 2009). Standardised rules exist in sport in order to ensure fairness. While 'natural' inequalities such as differences in physiological and psychological make-up are tested through sport, artificial inequalities are seen as undesirable and often limited, as described in the previous chapter with regard to the availability of full-body swimsuits. However, while an issue such as a swimsuit was easily solved owing to its agreed-upon artificiality, the question of altitude was far more complex.

On the one hand, there were athletes whose actor-network included already living at high altitude and were therefore already adapted, giving them an advantage at the Mexico games. But their physiological make-up as a result of living there could be seen as 'natural' and therefore impossible and unnecessary to 
regulate. The IOC also suspected that wealthier teams might spend time living at altitude prior to the games in order to gain an advantage. But it was deemed too difficult and not appropriate to segregate competitions into high- and low-living athletes. Therefore, in order to attain fairness, the IOC issued the following statement:

No athlete other than those who usually live and train at such heights shall specially do so more than 4 weeks in the last 3 months before the opening of the Games. The IOC points out that to break this rule would be a gross breach of good sportsmanship and it is sure that no-one connected with the Olympic Movement would wish in any way to be guilty of taking advantage over the other competitors. (IOC, n.d., cited in Kasperowski, 2009, p. 1269)

While this statement was perceived to solve the problem in terms of fairness, national teams were still concerned about the effects of altitude on their athletes, with several nations sending scientists to study the effects of altitude. Through a study of Belgian athletes it was noted that athletes who trained at altitude often performed better once they returned to sea-level. This notion soon became commonplace, through the concept of 'living high, training low', developed as a way for athletes to gain the benefits from altitude training while still training at normal intensity (Kasperowski, 2009; Levine, 2006).

Bowers (2009) describes the principle behind altitude training. At altitude the body increases its oxygen-carrying capacity (through producing erythropoietin $(\mathrm{EPO}))$ in response to the low-oxygen environment that occurs at high altitude. However, this effect only lasts for a short duration, with EPO production peaking at about forty-eight hours of altitude acclimatisation, and base-lining again after five to ten days. Furthermore, training intensity is negatively affected by altitude. Therefore, through 'living high, training low' an athlete is able to maximise their oxygen intake while still ensuring maximum training intensity. However, the problem of training this way is gaining access to mountains and valleys that allow athletes to move from high to low altitude while still allowing-high intensity training.

TCHEs were developed as an attempt to mirror the effects of living high and training low. Levine (2006, p. 297) describes how

Heikki Rusko ... decided to use an old industrial technique designed to filter oxygen molecules and dilute a room with nitrogen to create a hypoxic environment similar to the mountains. Athletes could then live and sleep in the 'nitrogen house' and train at sea level. 
There are now various versions of these environments available, consisting of either tent-like structures, smaller sleeping-bag-like tubes or devices that control the air in a single room (Bowers, 2009).

However, the outcomes from using these devices remain unclear (Bowers, 2009; Levine, 2006). Study design differences have complicated the issue, and while studies measuring simply EPO production have consistently recorded a modest increase, whether this translates into increased performance is debatable (Bowers, 2009). For example, in one study it is argued that 'the physiological response to altitude is quite variable and unpredictable among individual athletes - for example, the increase in EPO levels at an equivalent altitude of $3000 \mathrm{~m}$ may range from a 400 per cent increase to a 40 per cent decrease after 24 h' (Ri-Li et al., 2002, cited in Levine, 2006, p. 298; italics in Levine). TCHEs are quite unusual in having such uncertainty, and therefore the general rule of thumb appears to be that each athlete must determine individually whether it is effective for them. For example, a marathon runner's blog records how her husband found them effective for his training, but that she personally had not found them useful, and instead experienced breathing difficulties (Herron, 2011).

\section{Should altitude chambers be permitted?}

Several philosophers and sports scientists have used a number of different criteria to examine the acceptability of TCHEs (see, for example, Fricker, 2005; Levine, 2006; Loland and Caplan, 2008; Miah, 2006). Fricker (2005) simply states that, as TCHEs do not alter the athlete in a superhuman way or cause harm, there is no ethical problem associated with them. In a more detailed analysis, Levine (2006) analyses WADA's decision not to ban TCHEs using the WADA criteria of whether they are performance-enhancing, whether they are safe and whether they are in the spirit of sport. WADA found that they could be performance-enhancing, therefore violating the spirit of sport, but they are also safe (Levine, 2006). In his article, Levine (2006) argued against two of their conclusions. First, he asserted that the potential for TCHEs to enhance performance is by no means certain since, as described above, the reactions of an individual are unpredictable. Second, he argues against the reasoning that TCHEs are against the spirit of sport on the grounds of 'passivity' in that they do not require active training to use, as WADA claims. He explains that the science of athlete 
recovery is an important part of training, despite it being passive, that many other passive actions in sport are acceptable and, finally, that TCHEs should be allowed, based on the fact that they simulate living at altitude, which is also acceptable. Miah (2006) agrees with Levine (2006) that the passivity argument is illogical, also pointing to other examples of passivity, including the role of the athlete in accepting advice from a coach. Miah (2006) further argues that TCHEs do not affect the doing of sport but may potentially contribute to the development of sport, with technologies increasingly being integral to improvements and developments in sporting performance, and for these reasons sees it as illogical to ban TCHEs.

Loland and Caplan (2008) evaluate TCHEs against the criteria of benefit, safeness and the possibility of providing fairness to determine whether they align with the spirit of sport. They conclude that the answer is ambiguous, stating that the benefits are clear - they are safe - but the last criterion depends on context. They argue that if TCHEs are used by athletes to help them adjust to altitude, then this is acceptable, but if TCHEs are used purely in an attempt to increase the oxygen-carrying capacity of the blood, this goes against the spirit of sport.

\section{The ANT perspective on why TCHEs can be viewed as acceptable}

In contrast to the arguments discussed above, the TCHE will now be considered as an actor-network, with distinctive ideas folded within it that make the decision to allow TCHEs potentially understandable. It is argued that because the actor-network that makes up the TCHE is very different from the actor-networks of doping and the use of other banned substances, TCHEs can be understood as natural and unproblematic.

First, the TCHE is designed, first and foremost, to simulate altitude training. Altitude training is indisputably allowed, given that the actor-network of some athletes includes living at higher altitudes. As Levine (2006, p. 300) points out: 'there is appropriately no movement to regulate where an athlete can live. That is, an athlete can choose to live wherever on the planet he or she can, regardless of the barometric pressure of the environment.' Therefore, TCHEs have the idea folded within them that they have the same effect for an athlete as happening to be living at high altitude. 
Altitude training is further understood as acceptable owing to the popular discourse that one of the reasons that long-distance runners from nations such as Kenya and Ethiopia have been so successful is that they live at higher than normal altitudes (Bale and Sang, 1996). In their intensive study of Kenyan runners, Bale and Sang discuss how the association between Kenyas high altitude and their success in running has led to the assumption that there is a causal relationship between the two. They describe a long history of environmental determinism tied to racist beliefs which has led to this discourse, but argue there is no conclusive evidence of it being accurate. They also point out that athletes who live at altitude in other areas do not necessarily succeed at long-distance running, and instead describe the intensive training performed by Kenyan runners, which, they argue, has had the greatest impact on their success. Nonetheless, despite the lack of evidence, the belief that athletes who live at higher altitudes are more successful remains a part of the understanding of why TCHEs are an acceptable training method.

The understanding of TCHEs as producing 'natural' bodily effects is confirmed through their link with the high-profile banned drug artificial EPO, which is now, particularly after the 2012 Lance Armstrong scandal, a well-known 'evil' in the study of sport. On one hand, TCHEs mirror the effects of taking artificial EPO since the goal of TCHEs is to increase the body's production of EPO. However the contrast between artificial EPO and using a TCHE is marked. Artificial EPO causes the body to change instantly, which can be viewed as a very unnatural response, while TCHEs allow the body to adapt more gradually, as it would do 'naturally'.

The problem is the additional actant of the TCHE, which acts as an intermediary in ensuring that the actor-network that makes up the inner workings of the body changes to include a higher production of EPO. But if we only consider the effects on the body, we can see how using a TCHE mirrors the bodily response of someone living at altitude and not the response of someone using artificial EPO.

A rather different component folded within the TCHE's actor-network is the price. TCHEs can be very expensive, and therefore only available to athletes with significant resources at their disposal. For example, while some simple versions of 'altitude tents' are easily available over the internet for only hundreds of dollars, these have the drawbacks of being hot and humid and increasing the production of $\mathrm{CO}^{2}$, which undermines the effect of the tent. Acquiring a device that overcomes these barriers can run to US $\$ 100,000$ (pers. comm. Michael Hamlin, 2013). These costs mean that it is only athletes or nations who are prepared to 
invest heavily in elite sport, and therefore those who commonly sit at the top of the medal table, who can afford these devices. Beamish and Ritchie (2006) describe how inequalities produced by performance-enhancing substances are very often the same inequalities as produced within sport more generally. It is the same with TCHEs. However, in this case the inequality can be rationalised through the idea that using these devices enables a less talented runner to reach the same level as runners from Kenya or Ethiopia, who are assumed to have these abilities naturally because they live at higher altitudes. Further, the discourse that African runners have a natural advantage owing to altitude simulates the historical ideas embedded in the concept of race that assume a natural advantage to all dark-skinned athletes. In this way, TCHEs and altitude training confirm normalised ideas surrounding inequalities in sport, confirming that 'white' athletes can gain success through hard training and access to resources such as TCHEs, while 'black' athletes are naturally superior.

There is, further, little concern about inequality in terms of availability because, unlike the swimsuits discussed in the previous chapter, the use of these devices is relatively invisible. A swimsuit is easily visible to all watching the race, but training, and particularly sleeping, are private activities not usually seen by outsiders or other athletes. This also connects to a point made by Levine (2006), who argued that, if TCHEs were banned, policing their use would be extremely difficult owing to the invisibility of their use, so would have to rely upon the creation of a culture of spying.

Finally, the behaviours involved in using the devices are not questionable in the way that, for example, doping is. Using TCHEs involves breathing in a different environment. It involves either a plastic tent or a sleeping-bag-like tube, which the athlete uses to perform activities such as sleeping or resting. The actor-network only includes very standard behaviour. This is quite different from an activity such as doping, which has connotations of deviance and anti-social behaviour (Miah, 2006) owing to the similarities to recreational drug-taking, and includes questionable devices such as syringes as part of its actor-network. Doping also can involve the introduction of artificial substances into the body, whereas TCHEs do not (Miah, 2006).

In sum, TCHEs imitate natural processes so effectively that their very artificiality almost becomes irrelevant. They produce the same effects as natural altitude training, including the same gradual change process within the body. The process of using TCHEs also encompasses only 'natural' behaviours. Athletes are able to live their normal lives, apart from breathing in a different environment for some part of the day. 
This example highlights the importance of understanding athletes as cyborgs, or as network assemblages made up of human and non-human elements. The effects of the TCHEs are so well designed to imitate the natural that they have less artificial effect than a runner wearing shoes. The assemblage of the human athlete with a TCHE produces the same effects as a human athlete living in a particular natural environment. With this in mind, the only reason to suggest that TCHEs should be on the banned list is that they are artificial. But if we dispense with the understanding that an athlete is an entirely natural entity and understand that, as a network assemblage, an athlete exists as a network of human and non-human, then TCHEs can be seen as entirely acceptable. This is a similar scenario to the case of Oscar Pistorius's legs, which are artificial but designed to imitate human legs so effectively that several years of examination could not determine any discernible difference. In both cases, the technologies act as innocuous parts of the athlete's network in the same way as running shoes, and it is only that they have the concept of artificiality folded within them that makes them problematic.

\section{Non-enrolment of TCHEs by athletes}

Despite the above, which can be seen as an explanation for why WADA has chosen not to ban TCHEs, this does not mean that they are always easily enrolled by athletes. The above description followed the actor-network of a TCHE in itself. But when TCHEs are utilised by athletes, the actor-network grows to include the athletes' own actor-network, which does not necessarily allow the two actants to assemble easily together.

An obvious reason for not enrolling TCHEs is, as described above, cost. Not all athletes or nations can afford them. Moreover, those nations that can afford them must find a way to provide access to all athletes. For example, nations that adopt a more centralised training model, where all athletes are concentrated together in one area surrounded by sports science support, would be more likely to find it easier to provide athletes with TCHEs than those who adopt a more independent model, in which, athletes are more likely to be scattered throughout the country, making it difficult to site a TCHE in a place which would benefit all athletes equally.

Also described earlier was the doubt surrounding the effectiveness of TCHEs. While most studies reveal that they generally increase EPO, it is not clear that this always leads to an increase in performance. Therefore, athletes are unlikely to adopt a practice where the benefits are unclear. 
There is also the question of whether enrolling the device will actually be of use to that particular athlete. One goal of the production of new technologies can be to overcome obstacles. From an ANT perspective, this is described as the creation of a programme to overcome an anti-programme (Latour, 1991). For example, in the sport of mountaineering, the obstacle of 'lack of oxygen' is overcome through the use of oxygen canisters which can be carried up the mountain (van Hilvoorde, Vos and de Wert, 2007, p. 175). In this example, 'lack of oxygen' acts as an anti-programme as it is a factor that makes the climbing of the mountain very difficult. Adding the technological device of the oxygen canister to the actor-network of the mountaineer overcomes the anti-programme and improves the chances of mountaineer reaching the top of the mountain.

For some athletes the TCHE does not overcome any obstacles because it is not present in their particular case. This could take the form of athletes already residing at altitude, or having greater genetic capacity to carry oxygen as part of their actor-network.

Finally, the ANT approach points out that all aspects of the network are connected. So if one aspect of the network is altered, some other part of training may also not work so well. For example, in distance runner Herron's (2011) online blog, she described how using a TCHE made the rest of her training regime less effective making her less inclined to adopt the practice. This is similar to the argument made in the previous chapter where the kayakers identified other parts of the network that were as important as the kayak itself.

As with the kayak designs discussed in the previous chapter, the non-enrolment of TCHEs calls into question the assumption that athletes are always seeking to improve their performance through taking on new technological developments. Instead, athletes' own individual genetic capacities and beliefs in what works for them are important in determining whether an athlete will enrol a new technology. Thus, the concern that natural sporting competitions may be ruined by the increasing inclusion of technologies appears to be unfounded.

\section{Conclusion}

This chapter considered the integration of 'foreign' technologies into sport through the cases of GPS in AFL and TCHEs. Both GPS and the TCHEs make interesting cases for study since their effectiveness has been questioned by researchers in both cases. With GPS, researchers suggest that the units are not 
used to full capacity. However, the devices are used to survey players, which players perceive to be problematic. With TCHEs, there is inconclusive evidence regarding their ability to improve performance. This chapter describes how other aspects of the socio-technical network have a strong influence on decisions surrounding the use of both technologies.

In both cases, I examined the technology as an actor-network and examined its enrolment and use through following the network. In the case of the GPS units, I followed the network outwards from the individual box and determined that there were other important parts of the network that were necessary for the GPS units to be used effectively which had not been considered by the league in their initial introduction. The league's lack of consideration of the GPS units as networks led to the units not being used to their full potential.

Nonetheless the process revealed two dominant reasons why the units have become common practice in the AFL. First, GPS units provide sufficient data to be useful to coaches and scientists. Second, GPS offers a level of surveillance that is very useful to coaches and managers, who want their teams to perform at their best, and so the ability to monitor players to ensure all are contributing to this goal is highly attractive. Both these reasons indicate that coaches and managers are more likely to make the decision to enrol GPS than players. However, the process also found that, becuase the league did not consider the need for a) other intermediaries to make the GPS work and b) ways of overcoming mediators such as roofs, the GPS units were not providing the level of value that could do.

Following the case of the TCHEs revealed a similar issue with the devices not providing value for all athletes. In this case, the lack of value was simply due to the fact that the way the TCHE assembles with each athlete is different in every case (which is not the case with the GPS units, which work with all athletes in the same way). The case of the TCHEs particularly emphasises the importance of understanding athletes as networks of human and non-human, or as cyborgs, rather than viewing new technologies as intrusions on a natural body. TCHEs have been designed to imitate natural processes so effectively that their artificiality becomes irrelevant, and so they should not be subject to the same scrutiny as practices such as doping, which will be considered in the next chapter.

Both cases illustrate the importance of understanding technologies as heterogeneous networks. Purely examining their material value and seeing them as isolated physical artefacts prevents an understanding of how they can be used 
within sport. A similar argument is made by Magdalinski (2009), who argues for the importance of understanding the cultural meaning of technologies within the sporting arena. However, ANT takes this notion further by arguing that meaning, history, physical materiality and combining with the athlete are also significant in understanding how and why technologies are utilised and regulated within sport. 